\title{
Ultracompact 160 Gbaud all-optical demultiplexing exploiting slow light in an engineered silicon photonic crystal waveguide
}

\author{
Bill Corcoran, ${ }^{1, *}$ Mark D. Pelusi, ${ }^{1}$ Christelle Monat, ${ }^{1}$ Juntao Li, ${ }^{2}$ Liam O’Faolain, ${ }^{2}$ \\ Thomas F. Krauss, ${ }^{2}$ and Benjamin J. Eggleton ${ }^{1}$ \\ ${ }^{1}$ Centre for Ultrahigh Bandwidth Devices for Optical Systems (CUDOS), Institute of Photonics Optical Sciences (IPOS), \\ School of Physics, The University of Sydney, Sydney, New South Wales 2006, Australia \\ ${ }^{2}$ Scottish Universities Physics Alliance (SUPA), School of Physics Astronomy, \\ University of St Andrews, St Andrews, Fife KY16 9SS, UK \\ *Corresponding author: billc@physics.usyd.edu.au
}

Received January 19, 2011; accepted March 23, 2011; posted April 5, 2011 (Doc. ID 141304); published April 29, 2011

\begin{abstract}
We demonstrate all-optical demultiplexing of a high-bandwidth, time-division multiplexed $160 \mathrm{Gbit} / \mathrm{s}$ signal to $10 \mathrm{Gbit} / \mathrm{s}$ channels, exploiting slow light enhanced four-wave mixing in a dispersion engineered, $96 \mu \mathrm{m}$ long planar photonic crystal waveguide. We report error-free (bit error rate $<10^{-9}$ ) operation of all 16 demultiplexed channels, with a power penalty of $2.2-2.4 \mathrm{~dB}$, highlighting the potential of these structures as a platform for ultracompact all-optical nonlinear processes. (C) 2011 Optical Society of America
\end{abstract}

OCIS codes: $190.4360,230.5298$.

The manipulation of subpicosecond length optical pulses, of fundamental interest for monitoring or controlling fast interactions and for potential application in terabaud serial communication links [1], requires response times for devices faster than those achievable with current electronics (i.e., $>100 \mathrm{GHz}$ ). Ultrafast optical nonlinearities, which can respond on femtosecond time scales [2], provide a useful mechanism to handle these short pulses all-optically. One key functionality required to handle signals running at baud rates $>100$ Gbaud is the ability to switch out low-baud-rate channels from a high-baud-rate data stream, i.e., demultiplexing of timedivision multiplexed data. There have been many successful demonstrations of time-division demultiplexing of signals exceeding $100 \mathrm{Gbaud}$, utilizing hundreds of meters of specialty fiber $[3,4]$ or centimeter length scale waveguides in periodically poled lithium niobate [5], semiconductor optical amplifier [6], chalcogenide [7]], or silicon/Si-hybrid [8-10] materials. Further increasing waveguide nonlinearity allows for a reduction in the length of such devices and would provide a path to dense integration of all-optical signal processing devices on a single photonic chip. The key to increasing the waveguide nonlinearity is maximizing the energy density within waveguides. A first step toward this is to utilize high-index contrast structures that are able to provide submicrometer confinement of light. To increase energy density further, resonant structures can be used, such as ring resonators [11] or photonic crystal $(\mathrm{PhC})$ cavities [12]. However, demonstrations utilizing these structures for all-optical switching have typically been limited in bandwidth from a few gigahertz to at most a few tens of gigahertz. Slow light in $\mathrm{PhC}$ waveguides provides a semiresonant method for enhancing nonlinear interactions through increased energy density with distributed feedback [13]. In contrast to resonant cavities, dispersion engineered $\mathrm{PhC}$ waveguides [14] have provided demonstrations of enhanced nonlinearities over almost a terahertz of bandwidth [15]. This has established the potential for these types of waveguides to provide lowfootprint, high-bandwidth all-optical devices.

Here we present $160 \mathrm{~Gb} / \mathrm{s}$ to $10 \mathrm{~Gb} / \mathrm{s}$ optical timedivision demultiplexing exploiting slow light enhanced nonlinearity in a $96 \mu \mathrm{m}$ long waveguide. To the best of our knowledge, this is the shortest device successfully used for demultiplexing of data streams above $100 \mathrm{GBaud}$, demonstrating the potential of dispersion engineered $\mathrm{PhC}$ waveguides in providing dense integration of all-optical elements on a chip-based platform.

Our demultiplexing scheme switches out $10 \mathrm{~Gb} / \mathrm{s}$ channels from a $160 \mathrm{~Gb} / \mathrm{s}$ data stream via four-wave mixing (FWM; Fig. 1) [9]. A $10 \mathrm{GHz}$ pulse train (at $1553.5 \mathrm{~nm}$ ), synchronized to the data stream (at $1547.5 \mathrm{~nm}$ ), converts a $10 \mathrm{~Gb} / \mathrm{s}$ channel in the $160 \mathrm{~Gb} / \mathrm{s}$ optical time-division multiplexed (OTDM) data stream to the FWM idler wavelength (around $1559.5 \mathrm{~nm}$ ). This demultiplexed channel can then be optically filtered to separate it from the $160 \mathrm{Gbit} / \mathrm{s}$ data stream.

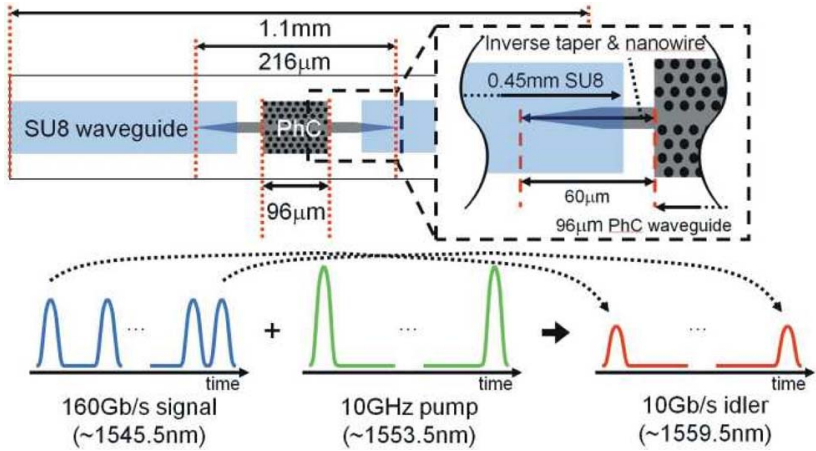

Fig. 1. (Color online) Top: device layout. The $96 \mu \mathrm{m} \mathrm{PhC}$ is connected to a $10 \mu \mathrm{m}$ long nanowire and $50 \mu \mathrm{m}$ long inverse taper. Light is coupled to the silicon waveguide segment via a polymer rib waveguide. Bottom: schematic of FWM demultiplexing. When the signal and pump temporally overlap, the signal is reproduced at the FWM idler wavelength. 
Slow light in the $96 \mu \mathrm{m}$ long $\mathrm{PhC}$ waveguide is crucial to achieving this functionality in our compact waveguide, as this enhances the FWM interaction. In the PhC waveguide, the FWM conversion efficiency is enhanced proportional to the product of the group indices of the four waves [16], i.e., scaling with $n_{g \text { pump }}^{2} \times n_{g \text { signal }} \times$ $n_{g \text { idler }}$. If all four waves involved travel at similar group velocities, the conversion efficiency of FWM is enhanced as $S^{4}$ (the slow down factor [17-19] $S=n_{g} / n_{\text {eff }} \sim 10 \pm$ $5 \%$ from 1545 to $1561 \mathrm{~nm}$ ). This enhancement can be used to lower the overall length of the waveguide needed to observe significant FWM $[\underline{18}, \underline{19}]$.

The device used in this demonstration (Fig. 1) incorporates a $96 \mu \mathrm{m}$ long dispersion engineered $\mathrm{PhC}$ waveguide [14]: an air-suspended silicon structure $220 \mathrm{~nm}$ thick, with a lattice period of $404 \mathrm{~nm}$, hole radii of $\sim 230 \mathrm{~nm}$, and row shift parameters [14] $s_{1}=-50 \mathrm{~nm}$ and $s_{2}=$ $0 \mathrm{~nm}$ (similar to that used to investigate FWM in [18]). It has a low dispersion region (see Fig. 3) with a group velocity of $v_{g} \sim c / 30$ over a $16 \mathrm{~nm}$ bandwidth from 1545 to $1561 \mathrm{~nm}$, with a modal effective area $\left(A_{\text {eff }} \sim 0.5 \mu \mathrm{m}^{2}\right)$. Coupling to the slow mode is enhanced by a mode conversion interface [20], and recently experiments have shown that similar dispersion engineered $\mathrm{PhC}$ waveguide designs provide record low propagation losses in the slow light regime [21]. The $\mathrm{PhC}$ waveguide is connected on both sides to $10 \mu \mathrm{m}$ long access nanowires $(220 \mathrm{~nm} \times 700 \mathrm{~nm}$ cross section) with $50 \mu \mathrm{m}$ long inverse tapers. Large mode area $\left(A_{\text {eff }} \sim 8 \mu \mathrm{m}^{2}\right)$ polymer waveguides complete the structure at both chip ends [19] to improve coupling to the device via butt-coupled lensed fibers (Fig. 1). The total fiber-to-fiber loss when probing the slow light band was $\sim 14 \mathrm{~dB}$, including losses (coupling and propagation) over the silicon waveguide segment of $\sim 3 \mathrm{~dB}$.

The signal is generated from a $40 \mathrm{GHz}$ optical pulse train (CLK), on-off keyed with a $2^{31}-1$ pattern length pseudorandom bit sequence (PRBS) at $40 \mathrm{~Gb} / \mathrm{s}$, then time interleaved to provide a $20 \%$ duty cycle, $160 \mathrm{~Gb} / \mathrm{s}$ signal. The pump is a $10 \mathrm{GHz}, 1.9 \%$ duty cycle pulse train. Both pump and signal are amplified, filtered, and combined immediately before to the silicon chip. The signal and pump are copolarized and coupled to the TE-like mode of the PhC waveguide. Dispersive broadening of the signal is minimized with a spectral phase filter (Finisar WaveShaper). Temporal alignment of the signal and pump pulses is ensured by maximizing the FWM idler at the output of the waveguide, controlled by a variable optical delay line. We estimate coupled average powers in the $\mathrm{PhC}$ waveguide of $\sim 17 \mathrm{~mW}$ and $\sim 14 \mathrm{~mW}$ for the pump and signal, respectively, with pump pulse energy at the beginning of the $\mathrm{PhC}$ waveguide $\sim 2.5 \mathrm{pJ}$. The demultiplexed channel at the idler frequency is passed through a $2 \mathrm{~nm}$ filter (centered $\sim 1559.5 \mathrm{~nm}$ ) before being sent to the receiver assembly (Fig. 2).

On chip, the FWM interaction used for demultiplexing occurs primarily in the $96 \mu \mathrm{m}$ long section of slow light $\mathrm{PhC}$ waveguide. The short length of the nanowire waveguides (see [18]) and the large mode area of the polymer waveguides limits FWM in those sections. By comparing the signal and idler powers, derived by integrating appropriate sections of the measured spectrum at the output of the waveguide [Fig. 3(b)], we infer an FWM conver-

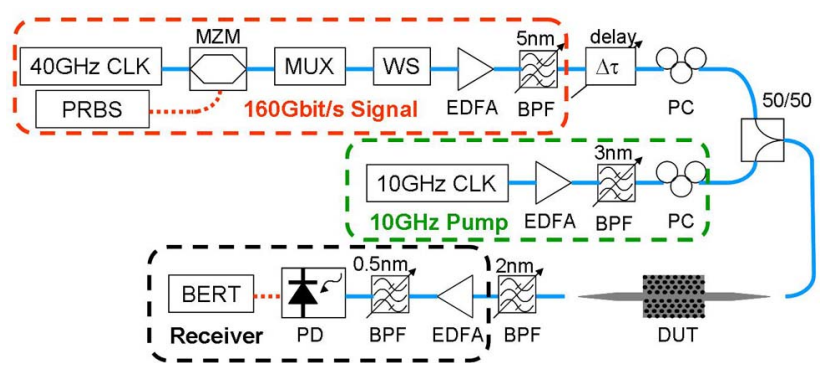

Fig. 2. (Color online) FWM demux experiment. A $40 \mathrm{GHz}$ pulse train is modulated (MZM) and time interleaved (MUX) to provide a $160 \mathrm{~Gb} / \mathrm{s}$ signal and copropagated with a $10 \mathrm{GHz}$ pulse train in the device under test (DUT) (WS-spectral phase filter, EDFA — erbium fiber amplifier, $\mathrm{BPF}$ - bandpass filter, $\mathrm{PC}$-polarization controller, PD—photodetector, BERT-bit error rate test).

sion efficiency $\left(\mathrm{CE}=P_{\text {idler out }} / P_{\text {signal out }}\right)$ of $\sim-33 \mathrm{~dB}$ $(\sim-18 \mathrm{~dB}$ instantaneous $\mathrm{CE}$, when taking into account a factor of $1 / 16$ for difference in repetition rate and $1 / 2$ for the $50 / 50$ data duty cycle PRBS). To quantify the enhancement of FWM afforded by slow light in our sample, we compare the measured value of instantaneous CE with an approximate calculated CE for an ideal $\mathrm{PhC}$ without the influence of slow light. Assuming lossless and dispersionless propagation, for a mode far from the band edge (typically $A_{\text {eff }} \sim 0.2 \mu \mathrm{m}^{2}, n_{g} \sim 6$ ), the CE can be calculated as $\left(\gamma P_{\text {peak pump }} L\right)^{2}=\left(\left[2 \pi n_{2}\right] /\right.$ $\left.\left[\lambda A_{\text {eff }}\right] P_{\text {peak pump }} L\right)^{2} \sim-41 \mathrm{~dB} \quad[\underline{18}]$, a factor of $\sim 200$ $(23 \mathrm{~dB})$ lower than the measured $\mathrm{CE}$. This difference in CE is primarily due to the different group index, effective area (CE scales as $S^{4}$ and as $1 / A_{\text {eff }}^{2}[16,18]$ ), and loss in the two cases compared and is lower than a simple factor of $S^{4} \sim 10^{4}$. We expect phase matching in these short waveguides to have a minor effect on CE [18]. Eye diagrams of initial $160 \mathrm{~Gb} / \mathrm{s}$ signal and demultiplexed $10 \mathrm{~Gb} / \mathrm{s}$ idler are measured [Figs. 4(a) and 4(b)], the idler clearly showing a single $10 \mathrm{~Gb} / \mathrm{s}$ channel with negligible residual traces of the other channels from the $160 \mathrm{~Gb} / \mathrm{s}$ data stream. By tuning the variable optical delay, all $1610 \mathrm{~Gb} / \mathrm{s}$ channels were demultiplexed and bit error rate (BER) curves measured.

The small spread in the demultiplexed channel BER curves indicates that the $160 \mathrm{Gbit} / \mathrm{s}$ signal was well multiplexed. The curves for the demultiplexed signals can be compared to the "back-to-back" case, where the pulses

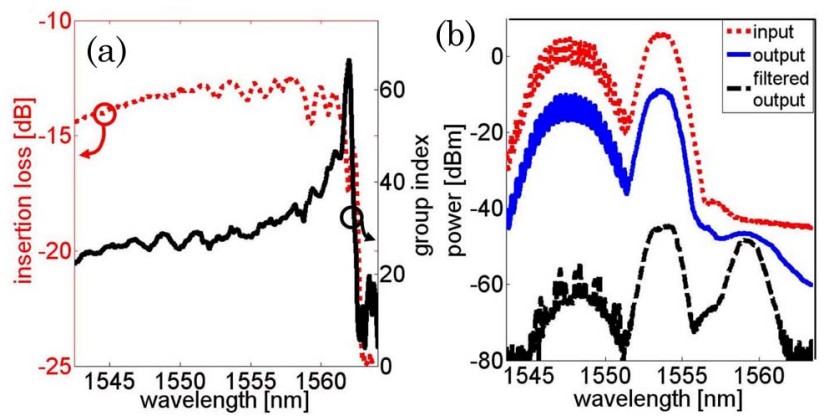

Fig. 3. (Color online) (a) Device insertion loss and PhC TE mode group index, (b) spectra captured by optical spectrum analyzer, pump $\sim 1553.5 \mathrm{~nm}$, signal $\sim 1547.5 \mathrm{~nm}$, generated idler $\sim 1559.5 \mathrm{~nm}$. The filtered output spectrum is after a $2 \mathrm{~nm}$ filter centered on the idler. 


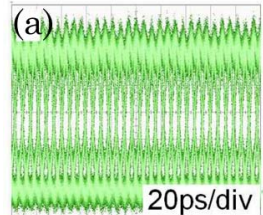

(b)
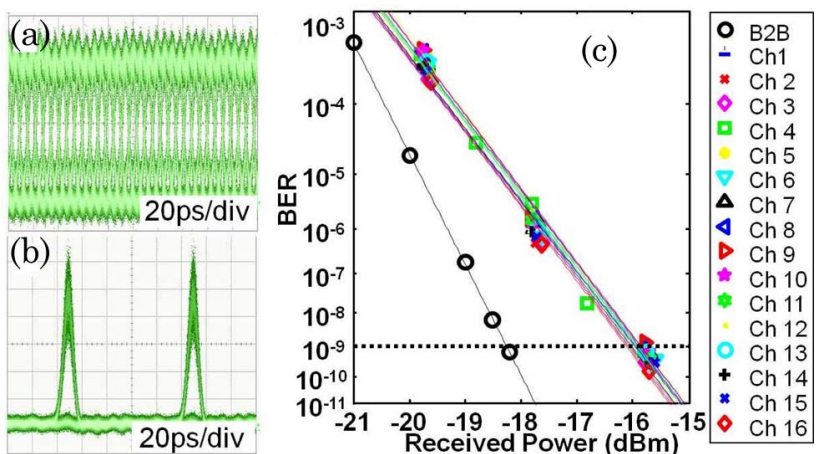

Fig. 4. (Color online) (a) $160 \mathrm{~Gb} / \mathrm{s}$ signal and (b) $10 \mathrm{~Gb} / \mathrm{s}$ idler eye diagrams from a $65 \mathrm{GHz}$ oscilloscope; (c) BER curves for all $1610 \mathrm{~Gb} / \mathrm{s}$ channels. "B2B" represents the back-to-back measurement.

from the $10 \mathrm{GHz}$ optical clock (previously used as the pump, now tuned to the idler wavelength) are modulated in the same manner as the signal, then bypass the $\mathrm{PhC}$ device to directly connect to the receiver assembly. Comparing the back-to-back BER curve with the demultiplexed curves, a power penalty of $2.2-2.4 \mathrm{~dB}$ is observed at the "error-free" (BER $<10^{-9}$ ) level. This penalty from our micrometer-scale device compares well with a recent similar demultiplexing experiment $(\sim 3.9 \mathrm{~dB}$ penalty) in a $1.1 \mathrm{~cm}$ long silicon nanowire waveguide [9]. It should be noted that the CE of our device was limited by nonlinear loss, which is significant in silicon waveguides. We would expect improved CE at lower pump powers by using longer $\mathrm{PhC}$ waveguides engineered for low linear propagation loss [21], which would lead to more efficient demultiplexing. Dispersion engineered structures in materials such as optimized IIIV semiconductor blends [22] or chalcogenide glasses [23] may also provide more efficient device operation.

In conclusion, we have successfully demonstrated error-free demultiplexing of $160 \mathrm{~Gb} / \mathrm{s}$ data in an ultracompact silicon PhC waveguide device. As the $96 \mu \mathrm{m}$ long $\mathrm{PhC}$ waveguide is the main nonlinear waveguide element on the chip, this shows the potential of such structures to play a key role in achieving dense integration of alloptical devices on a single photonic chip.

This work was supported in part by the Centres of Excellence and Federation Fellowship programs of the Australian Research Council. The silicon waveguide chip was fabricated under the Engineering and Physical Sciences Research Council-UK silicon photonics consortium and supported by the European Union (EU) Seventh Framework Programme Marie Curie project "OSIRIS."

\section{References}

1. H. C. H. Mulvad, L. K. Oxenløwe, M. Galili, A. T. Clausen, L. Gruner-Nielsen, and P. Jeppesen, Electron. Lett. 45, 280 (2009).

2. R. W. Boyd, Nonlinear Optics, 3rd ed. (Academic, 2008).

3. S. Kawanishi, H. Takara, T. Morioka, O. Kamatani, and M. Saruwatari, Electron. Lett. 31, 816 (1995).

4. H. Hu, H. C. H. Mulvad, M. Galili, E. Palushani, J. Xu, A. T. Clausen, L. K. Oxenløwe, and P. Jeppesen, J. Lightwave Technol. 28, 1789 (2010).

5. F. Gomez-Agis, C. Okonkwo, A. Albores-Mejia, E. Tangdiongga, and H. Dorren, Electron. Lett. 46, 1008 (2010).

6. E. Tangdiongga, Y. Liu, H. de Waardt, G. D. Khoe, A. M. J. Koonen, H. J. S. Dorren, X. Shu, and I. Bennion, Opt. Lett. 32, 835 (2007).

7. M. Galili, J. Xu, H. C. Mulvad, L. K. Oxenløwe, A. T. Clausen, P. Jeppesen, B. Luther-Davies, S. Madden, A. Rode, D.-Y. Choi, M. Pelusi, F. Luan, and B. J. Eggleton, Opt. Express 17, 2182 (2009).

8. C. Koos, P. Vorreau, T. Vallaitis, P. Dumon, W. Bogaerts, R. Baets, B. Esembeson, I. Biaggio, T. Michinobu, F. Diederich, W. Freude, and J. Leuthold, Nat. Photon. 3, 216 (2009).

9. F. Li, M. Pelusi, D.-X. Xu, A. Densmore, R. Ma, S. Janz, and D. Moss, Opt. Express 18, 3905 (2010).

10. H. Ji, M. Galili, H. Hu, M. Pu, L. Oxenløwe, K. Yvind, J. Hvam, and P. Jeppesen, IEEE Photon. Technol. Lett. 22, 1762 (2010).

11. V. R. Almeida, C. A. Barrios, R. R. Panepucci, and M. Lipson, Nature 431, 1081 (2004).

12. K. Nozaki, T. Tanabe, A. Shinya, S. Matsuo, T. Sato, H. Taniyama, and M. Notomi, Nat. Photon. 4, 477 (2010).

13. T. Baba, Nat. Photon. 2, 465 (2008).

14. J. Li, T. P. White, L. O'Faolain, A. Gomez-Iglesias, and T. F. Krauss, Opt. Express 16, 6227 (2008).

15. B. Corcoran, C. Monat, M. Pelusi, C. Grillet, T. P. White, L. O'Faolain, T. F. Krauss, B. J. Eggleton, and D. J. Moss, Opt. Express 18, 7770 (2010).

16. M. Santagiustina, C. Someda, G. Vadala, S. Combrie, and A. De Rossi, Opt. Express 18, 21024 (2010).

17. T. F. Krauss, J. Phys. D 40, 2666 (2007).

18. C. Monat, M. Ebnali-Heidari, C. Grillet, B. Corcoran, B. J. Eggleton, T. P. White, L. O'Faolain, J. Li, and T. F. Krauss, Opt. Express 18, 22915 (2010).

19. J. Li, L. O'Faolain, I. H. Rey, and T. F. Krauss, Opt. Express 19, 4458 (2011)

20. S. Schulz, L. O'Faolain, D. Beggs, T. White, A. Melloni, and T. Krauss, J. Opt. 12, 104004 (2010).

21. L. O'Faolain, S. A. Schulz, D. M. Beggs, T. P. White, M. Spasenović, L. Kuipers, F. Morichetti, A. Melloni, S. Mazoyer, J. P. Hugonin, P. Lalanne, and T. F. Krauss, Opt. Express 18, 27627 (2010).

22. V. Eckhouse, I. Cestier, G. Eisenstein, S. Combrie, P. Colman, A. De Rossi, M. Santagiustina, C. G. Someda, and G. Vadala, Opt. Lett. 35, 1440 (2010).

23. K. Suzuki, Y. Hamachi, and T. Baba, Opt. Express 17, 22393 (2009). 